\title{
CHEMISTRY OF MUSCULAR CONTRACTION
}

$\mathrm{T}$ HE "Chemistry of Muscular Contraction" was discussed jointly by Sections B (Chemistry) and I (Physiology) of the British Association on September 6 , during the recent meeting at Birmingham.

Prof. Ernest Baldwin (University College, London) gave an introductory address, stressing the uncertainty of present views on muscle chemistry. He began with a brief historical review from the period beginning with Fletcher and Hopkins's classical experiments to the present day. The work of Fletcher and Hopkins provided the first unequivocal evidence that muscular activity is associated with the degradation of glycogen and the formation from it, under anaerobic conditions, of lactic acid. That glycolysis is not, however, the immediate energy source was discovered by Lundsgaard's demonstration of 'alactic acid' contraction in muscles poisoned with iodoacetate. The activity of iodoacetate-poisoned muscle is attended by the decomposition of phosphagen, glycolysis being totally abolished. Exhaustion of the supplies of phosphagen is promptly followed by rigor, and hence it follows that phosphagen breakdown is a more immediate source of energy than glycolysis.

Phosphagen breaks down rapidly if added to fresh muscle extracts prepared after the method of Meyerhof, but fails to decompose if added to exhaustively dialysed extracts. This, as Lohmann was able to show, was due to the absence from dialysed extracts of adenosine monophosphate. Subsequent work showed that, in fact, phosphagen (creatine phosphate) reacts with adenosinediphosphate to yigld adenosinetriphosphate and free creatine through the Lohmann reaction, a "phosphate-plus-energy transfer":

Creatine phosphate + adenosinediphosphate $\rightleftharpoons$ creatine + adenosinetriphosphate

The involvement of adenosinemonophosphate in Lohmann's original experiments was due to the intervention of another enzyme, myokinase.

Since resting muscle contains both adenosinetriphosphate and a powerful adenosinetriphosphatase, but does not contain the di- or mono-phosphate, it seems certain that, in alactic acid contraction as well as in unpoisoned muscle, the breakdown of phosphagen must necessarily be preceded by some breakdown of adenosinetriphosphate to yield the diphosphate. Phosphagen is not known to be broken down in any other way in muscle or in muscle extract, but only by interaction with adenosinediphosphate. Thus the decomposition of adenosinetriphosphate into the diphosphate, inorganic phosphate and energy assumed the position of the earliest known energyyielding reaction in extracts and probably, therefore, in muscle also.

For this there is at present no direct evidence, and it has not yet been possible to meet A. V. Hill's challenge to biochemists to produce such evidence, mainly because sufficiently refined methods of chemical analysis are not at present available.

The present phase of muscle chemistry began with Engelhart and Lubimova's demonstration, now amply confirmed, of the adenosinetriphosphatase nature of myosin, the predominant protein of muscle, and a protein, moreover, which is known to have contractile properties. Most early preparations of myosin were, however, more or less grossly contamin- ated with actomyosin, the product of interaction of myosin with a second muscle protein, actin. Acto. myosin consists of long, fibrous particles, and much recent research has amply demonstrated the fact that these particles undergo more or less extensive shortening if treated with adenosinetriphosphate, an effect which is exceedingly specific.

Such at present seems to be the physicochemical basis of muscular contraction. In relaxed muscle, both adenosinetriphosphate and actomyosin are present, but are apparently separated in some way until the arrival of a stimulus to contraction. When the two come together the fibres shorten, apparently undergoing dissociation to yield free actin and a myosin-adenosinetriphosphate complex. This latter is, in effect, an enzyme-substrate complex. Only now does adenosinetriphosphate undergo decomposition, yielding, so far as we know, adenosinediphosphate, inorganic phosphate and energy; myosin is regenerated and reacts again with the actin to re-form the longer threads of actomyosin. If this picture is correct, relaxation rather than contraction would have to be considered as the process in which adenosinetriphosphate is broken down, the energy arising from this breakdown being utilized to restore the contracted muscle to its relaxed configuration. The contracted condition, in fact, would seem to be the truly resting state of muscle; a state in which adenosinetriphosphate and actomyosin can perhaps exist as a temporarily stable intermediate reaction complex.

Prof. Baldwin sees in such a theory as this the beginnings of an understanding of the behaviour of the adductor muscles of lamellibranch molluscs, many of which can keep the shells of the animal closed for long periods of time and against relatively enormous loads, yet without any very appreciable consumption of energy. He suggested that more attention might now be profitably paid to these and similar unstriated muscles.

In conclusion, Prof. Baldwin remarked on the frequent revolutions that have already taken place in the field of muscle chemistry, and commented that yet another may be imminent. The picture he had given of the present position was, he pointed out, only one possible interpretation of the experimental facts-something in the nature of an "Aunt Sally", put up in the confident expectation that it would shortly be knocked down.

Dr. S. V. Perry (School of Biochemistry, Cambridge) presented a paper entitled "Adenosinetriphosphate and the Isolated Myofibril". He reminded the audience of the principal structural features of a typical, intact striated muscle. Normal methods of extraction with saline media of low and high ionic strengths extract the sarcoplasmic and myofibrillar constituents respectively, but entail complete structural disorganisation of the tissue. By the use of a collagenase obtained from Clostridium welchii, it has been possible, however, to disintegrate only the connective tissues of the muscle and obtain in this way a fine dispersion of intact myofibrils. 'Their detailed structure was illustrated by $\mathbf{a}$ beautiful electron micrograph which showed cross-striations corresponding elosely to those of the whole muscle. The micrograph revealed the existence of protein threads, presumably of actomyosin, running through 
out the length of the myofibril and apparently anchored at the $Z$ bands. These isolated myofibrils shorten to about 25 per cent of their initial length in the presence of $0.001 M$ magnesium ion and $0.0004 M$ adenosinetriphosphate. A further electron micrograph showed the concertina-like appearance of the shortened myofibril and the disappearance of most of the finer structures originally visible.

The myofibrils decompose adenosinetriphosphate; if they are not carefully prepared, the triphosphate breaks down as far as the monophosphate. Apparently this is due to the presence of myokinase, since repeatedly washed preparations carry the breakdown only as far as the diphosphate. If the muscle is put into rigor by freezing and thawing, there is gross disruption of the structure and normal myofibrils cannot be obtained. Muscles in rigor mortis do not undergo this disruption, and they yield good myofibril preparations. As Dr. Perry pointed out later, the decomposition of adenosinetriphosphate which occurs in rigor mortis, as opposed to that in freezethaw rigor, does not appear to be accompanied by the shortening of actomyosin, but may be due to the newly discovered adenosinetriphosphatase of Meyerhof, which appears to be localized in the sarcoplasm and of which the function is still obscure.

Dr. Perry went on to describe experiments which showed that the effects of magnesium and calcium ions on the adenosinetriphosphatase activity of isolated myofibrils differ in some respects from the effects obtained with extracted actomyosin. Isolated myofibrils also show differences from artificial threads made from actomyosin in their physical behaviour with adenosinetriphosphate; in particular, the shortening of actomyosin threads is essentially dehydrative and is isodimensional. That of the myofibrils, however, is longitudinal, with little or no lateral change, and thus more closely resembles normal muscle contraction. These differences, Dr. Perry believes, are due to differences in the structura] organisation of the two kinds of preparations. $\mathrm{He}$ then passed on to discuss possible relationships between the enzymic and structural organisation of the muscle cell on one hand and the phenomenon of cellular contractility on the other. In the main, these appear to be in agreement with the general picture previously given by Prof. Baldwin.

Dr. J. R. Bendall (Low Temperature Research Station, Cambridge), in a paper entitled "Shortening of Muscle in rigor mortis", chose to assume that the contraction of a muscle passing into rigor mortis can be considered as a very protracted and irreversible form of muscular contraction, though he was well aware of the dangers which this assumption implies. Mammalian (rabbit psoas) muscle going into rigor does not shorten much at room temperature, but is capable of doing a good deal of mechanical work at higher temperatures $\left(37^{\circ}\right)$ and especially at high $p H(7 \cdot 25)$. This shortening and work-performance are associated with three chemical phenomena known to occur in normal muscle, namely : (a) fall of $p \mathrm{H}$ due to lactic acid formation, which can be minimized by using starved or paralysed animals; $(b)$ breakdown of phosphagen; and (c) breakdown of adenosinetriphosphate.

A number of slides in which the magnitudes of these three phenomena were plotted against time, together with plots of the degree of shortening, all on a percentage basis of the initial values, were shown by Dr. Bendall. The rate of shortening and the amount of work done is higher at higher $p \mathrm{H}$, but neither the rate of $p \mathrm{H}$ change nor that of phosphagen breakdown parallels the rate of shortening. On the other hand, the breakdown of adenosinetriphosphate begins at about the same time as the onset of shortening, and the two processes are well correlated. The disappearance of phosphagen in spite of vigorous glycolysis is almost complete before adenosinetriphosphate breakdown assumes major proportions. Shortening is never found to begin before adenosinetriphosphate breakdown.

If Dr. Bendall's assumption be correct, his results would indicate that the breakdown of adenosinetriphosphate is associated with the phase of shortening rather than with that of elongation in normal muscle, a conclusion diametrically opposed to the scheme proposed by Prof. Baldwin. Dr. Bendall pointed out that his observations would account for the thermal data obtained by Prof. A. V. Hill if his initial assumption were true.

There were few comments and little discussion, apart from some observations on the properties and behaviour of artificial threads of actomyosin and myosin, which took place mainly between the speakers themselves. It appeared that the behaviour of actomyosin threads, free to shorten or placed under load, can be accounted for by the known properties of actomyosin, and that some important differences between these threads and isolated myofibrils can be attributed to differences in the orientation of the actomyosin fibres in the two cases. Prof. Baldwin, in concluding the discussion, said there had been much more agreement between himself and his fellow contributors than he had expected, and he was particularly interested in the contrasts between Dr. Perry's myofibrils and artificial actomyosin fibres of varying degrees of orientation.

\section{SCIENTIFIC INTERESTS OF ADULTS*}

\author{
By W. E. FLOOD \\ Education Department, University of Birmingham
}

$\mathrm{T}$ THE investigations described here form part of a wider study of the techniques of presenting science, by book or lecture, to the ordinary, nonacademic adult. Exploratory examinations were made of the relative interest in the various branches of science and of the relative appeal of different lines of approach to scientific topies.

A new classification of twenty-three science 'subjects' was devised for the investigation of interests. This included the recognized branches of science (for example, astronomy, geology) and also certain aspects of science such as industrial applications, new discoveries, scientific biography, and public direction and use of science. The interpretations to be placed on the twenty-three subject headings were briefly explained on the test-sheets used in the inquiries.

At this early stage of the study it seemed desirable to examine the preferences of adults who have revealed some definite interest in science. A testgroup of about four hundred adults (called Group I) was constructed from people voluntarily attending science lectures provided by the Workers' Educational Association (and a few by university extra-mural departments) in various parts of the country. They

* Substance of a paper read on September 1 before Section J (Psychology) of the British Association meeting at Birmingham. 